\title{
Association of polymorphisms hOGGI rs I052/33 and hMUTYH rs3219472 with risk of nasopharyngeal carcinoma in a Chinese population
}

This article was published in the following Dove Press journal:

OncoTargets and Therapy

I2 February 2016

Number of times this article has been viewed

Ying $X i e^{2}$

Yuan $\mathrm{Wu}^{3}$

Xunzhao Zhou ${ }^{3}$

Mengwei $\mathrm{Yao}^{3}$

Sisi Ning ${ }^{3}$

Zhengbo Wei

'Department of Head and Neck Tumor Surgery, Affiliated Tumor Hospital of Guangxi Medical University, ${ }^{2}$ Guangxi Key Laboratory for High-Incidence Tumor Prevention and Treatment, Experimental Center of Medical Science of Guangxi Medical University, ${ }^{3}$ Graduate School of Guangxi Medical University, Nanning, People's Republic of China
Correspondence: Zhengbo Wei Department of Head and Neck Tumor Surgery, Affiliated Tumor Hospital of Guangxi Medical University, 7I Hedi Road, 53002 I Nanning, People's

Republic of China

Tel +867715343612

Fax +86 7715303297

Emailwzhbo1973@aliyun.com
Abstract: This case-control study investigates the possible relationships between the single-nucleotide polymorphisms rs1052133 in the human 8-oxoguanine DNA glycosylase 1 (hOGGl) gene and rs3219472 in the human MutY glycosylase homologue (hMUTYH) gene and the risk of nasopharyngeal carcinoma (NPC). The two polymorphisms were genotyped in 488 unrelated NPC patients and 573 cancer-free controls. Genotype GG at rs 1052133 was associated with significantly lower NPC risk than genotypes GC + CC (odds ratio [OR] 0.770, 95\% confidence interval $[\mathrm{CI}] 0.595-0.996, P=0.012$ ). In subgroup analyses, subjects with genotype GG at rs 1052133 were at lower risk of NPC than those with GC or CC among individuals older than 40 years (OR $0.706,95 \%$ CI $0.524-0.950$ ), women (OR 0.571, 95\% CI 0.337-0.968), and those with no smoking history (OR $0.634,95 \%$ CI $0.463-0.868$ ). No significant association was seen between polymorphisms at $h M U T Y H \mathrm{rs} 3219472$ and the risk of NPC. However, gene-gene interaction analysis showed that subjects with genotype $\mathrm{CC}$ at rs1052133 and genotype AA at rs3219472 (CC/AA) were at 2.887-fold higher risk of NPC than those with GG/GG, 3.183fold higher risk than those with GG/GA, and 3.392-fold higher risk than those with GG/AA. Our results suggest that $h O G G 1$ rs 1052133 polymorphism may play an important role in NPC pathogenesis, especially among women, $>40$ years old, and those with no smoking history. The $h M U T Y H$ rs3219472 polymorphism may interact with $h O G G 1$ rs1052133 polymorphism to influence susceptibility to NPC.

Keywords: nasopharyngeal carcinoma, base excision repair pathway, human 8-oxoguanine DNA glycosylase 1, human MutY glycosylase homologue, single-nucleotide polymorphism

\section{Introduction}

Nasopharyngeal carcinoma (NPC) is a common malignant tumor in the southern part of People's Republic of China, including the provinces of Guangdong and Guangxi. In these areas, its incidence is up to 20-30/100,000 per year. $^{1}$ Mechanisms of NPC onset and progression remain unknown, though they are thought to be related to genomic DNA damage, as in many other malignant tumors. ${ }^{2,3}$ Oxidative DNA damage is one of the most frequent causes of malignant tumors, and 7,8-dihydro-8-oxoguanine is one of the best characterized oxidative DNA lesions. This lesion causes mutagenic transversion of $\mathrm{G}: \mathrm{C}$ to $\mathrm{T}: \mathrm{A}$ in several tumor suppressor genes and oncogenes, which can lead to carcinogenesis. ${ }^{4,5}$ 7,8-Dihydro-8-oxoguanine lesions are recognized and repaired effectively by the base excision repair (BER) pathway, in which 8-oxoguanine DNA glycosylase 1 and MutY glycosylase homologue (MUTYH) are the main enzymes. ${ }^{6}$

Single-nucleotide polymorphisms (SNPs) in key genes in the BER pathway, including the gene encoding human 8-oxoguanine DNA glycosylase 1 (hOGG1), have been 
associated with higher risk of many malignant tumors. ${ }^{7-9}$ Whether the same holds for NPC remains unclear, since studies looking for associations between hOGG1 SNPs and risk of NPC have given conflicting results. One study in Taiwan suggested that genotypes GG and GC at hOGG1 rs1052133 significantly increase the risk of NPC. ${ }^{10}$ However, studies of cohorts in Morocco, Algeria, Tunisia, and Chongqing in southwestern part of People's Republic of China suggested that hOGG1 rs1052133 polymorphism may not affect susceptibility to NPC. ${ }^{11,12}$

Similar to hOGG1, human MUTYH (hMUTYH) has been linked to cancer. Malfunction of hMUTYH has been associated with somatic transversion from G:C to T:A, potentially contributing to carcinogenesis. ${ }^{6}$ SNPs of MUTYH have been associated with susceptibility to malignant tumors. ${ }^{13}$ The hMUTYH rs3219472 SNP may influence the risk of several types of malignant tumor, such as esophageal adenocarcinoma and cholangiocarcinoma. ${ }^{14,15} \mathrm{We}$ are unaware of studies evaluating whether hMUTYH SNPs are associated with the risk of NPC.

To address these gaps in the literature, we conducted a case-control study involving 488 unrelated NPC cases and 573 cancer-free subjects. We examined possible relationships of hMUTYH rs3219472 and hOGG1 rs1052133 SNPs with the risk of NPC.

\section{Methods}

\section{Patients}

The present study included 488 unrelated NPC patients and 573 age- and sex-matched cancer-free subjects. Patients were diagnosed with NPC and treated at the Affiliated Tumor Hospital of Guangxi Medical University in Guangxi, People's Republic of China, between July 2012 and July 2014. This study was approved by the Ethics Committee of Guangxi Medical University, and informed written consent was obtained from all patients and healthy control subjects prior to sample collection.

\section{Blood sample collection}

Venous blood (3 mL) was collected from patients or healthy subjects in ethylenediaminetetraacetic acid-containing tubes for DNA extraction. Genomic DNA was extracted from blood using the TGuide Blood Genomic DNA Kit (Tiangen, Beijing, People's Republic of China), and DNA quality was assessed using agarose gel electrophoresis.

\section{Genotyping}

SNPs in genes encoding hOGG1 and hMUTYH were genotyped using the TaqMan real-time polymerase chain reaction (PCR) in a StepOnePlus ${ }^{\mathrm{TM}}$ System (Thermo
Fisher Scientific, Waltham, MA, USA) according to the manufacturer's instructions. Primers amplifying rs 1052133 SNP(C_3095552_1, Thermo Fisher Scientific) and rs3219472 (C_29736116_20, Thermo Fisher Scientific) were used in the PCRs. Each PCR $(10 \mu \mathrm{L})$ contained $5 \mu \mathrm{L} 2 \times$ TaqMan Master Mix, $0.5 \mu \mathrm{L}$ primers and probes, and $0.5 \mu \mathrm{L}$ DNA (15-25 ng/ $\mu L$ ). Reactions were run on 96-well Fast Plates (Thermo Fisher Scientific). Amplification was performed under the following cycling conditions: $95^{\circ} \mathrm{C}$ for 10 minutes, followed by 40 cycles of $95^{\circ} \mathrm{C}$ for 15 seconds and $60^{\circ} \mathrm{C}$ for 1 minute. Then the alleles in each reaction were determined using the TaqMan $^{\circledR}$ Genotyper ${ }^{\mathrm{TM}}$ software (Thermo Fisher Scientific). For quality control, 15\% samples were randomly selected and reanalyzed by a technician blinded to the sample identity. The results were identical to the original analysis.

\section{Statistical analysis}

Data were analyzed using Statistical Package for the Social Sciences 15 (IBM Corporation, Armonk, NY, USA). Genotype distribution among cancer-free subjects was assessed for Hardy-Weinberg equilibrium using Pearson's two-sided chi-squared test. Differences in genotype and allele frequencies between patients and healthy controls were assessed for significance using the chi-squared test. Correlation between NPC risk and particular genotypes was assessed using logistic regression to calculate crude odds ratios (ORs) with $95 \%$ confidence intervals (CIs). Adjusted ORs were calculated using multivariate logistic regression while controlling for various combinations of age, sex, and smoking history. A two-sided $P<0.05$ was defined as the threshold of significance.

\section{Results}

\section{Characteristics of patients and controls}

The genes hOGG1 rs1052133 and MUTYH rs3219472 SNPs were genotyped in all 488 patients with NPC and all 573 cancer-free controls (Table 1). The two groups showed no

Table I Clinical characteristics of Chinese patients with nasopharyngeal carcinoma and cancer-free controls

\begin{tabular}{llll}
\hline Characteristic & NPC & Control & P-value \\
\hline Sex, M/F & $374 / I 14$ & $417 / 156$ & 0.150 \\
Age (years) & $47.0 \pm 11.4$ & $47.9 \pm 12.5$ & 0.196 \\
EBV-VCA-IgA & & & \\
$\quad$ Positive & 304 & 8 & \\
$\quad$ Negative & 184 & 565 & $<0.00 \mathrm{I}$ \\
Smoking history & & & \\
$\quad$ Yes & 167 & 183 & 0.433 \\
No & 321 & 390 & \\
\hline
\end{tabular}

Note: Values shown are $n$ or mean \pm SD.

Abbreviations: NPC, nasopharyngeal carcinoma; M, male; F, female; EBV-VCA$\operatorname{lgA}$, IgA against the capsid antigen of Epstein-Barr virus; SD, standard deviation. 
Table 2 Genotype frequencies at hOGGI rsI052I33 and hMUTYH rs3219472 in Chinese patients with nasopharyngeal carcinoma and cancer-free controls

\begin{tabular}{|c|c|c|c|c|c|c|}
\hline \multirow[t]{2}{*}{ Polymorphism } & \multirow[t]{2}{*}{ Genotype } & \multicolumn{2}{|l|}{$\mathbf{N}(\%)$} & \multirow[t]{2}{*}{ OR (95\% Cl) } & \multirow[t]{2}{*}{ Adjusted OR ${ }^{a}(95 \% \mathrm{Cl})$} & \multirow[t]{2}{*}{$P_{\text {HWE }}$} \\
\hline & & NPC & Control & & & \\
\hline \multirow[t]{2}{*}{ rs 1052133} & $\mathrm{CC}+\mathrm{CG}$ & $337(69.1)$ & $362(63.2)$ & $0.769(0.595-0.993)$ & $0.770(0.595-0.996)$ & 0.930 \\
\hline & GG & $|5|(30.9)$ & $211(36.8)$ & & & \\
\hline \multirow[t]{2}{*}{ rs3219472 } & $\mathrm{GG}+\mathrm{GA}$ & $425(87.1)$ & $50 \mathrm{I}(87.4)$ & $0.969(0.675-1.392)$ & 0.971 (0.676-1.395) & 0.896 \\
\hline & $\mathrm{AA}$ & $63(12.9)$ & $72(12.6)$ & & & \\
\hline
\end{tabular}

Note: aCalculated by multiple logistic regression after controlling for age, sex, and smoking history.

Abbreviations: hOGGI, human 8-oxoguanine DNA glycosylase I; hMUTYH, human MutY glycosylase homologue; NPC, nasopharyngeal carcinoma; OR, odds ratio; $\mathrm{Cl}$, confidence interval; $\mathrm{HWE}$, Hardy-Weinberg equilibrium.

significant differences in age $(P=0.446)$, sex $(P=0.183)$, or smoking history $(P=0.433)$. In contrast, patients showed a significantly higher incidence of positive Epstein-Barr virus (EBV)-related antibody than the cancer-free group $(P<0.001)$. Genotype frequencies in the control group were consistent with Hardy-Weinberg equilibrium at both hOGG1 rs1052133 $(P=0.930)$ and hMUTYH rs3219472 ( $P=0.896$; Table 2$)$.

\section{Association of hOGGI rsI052/33 polymorphism with risk of NPC}

As shown in Table 2, the frequency of genotype GG at hOGG1 rs1052133 was significantly lower among patients than among controls (30.9\% vs 36.8\%), and the OR for NPC associated with genotype GG relative to NPC associated with genotypes CC or CG was 0.769 (95\% CI 0.595-0.993). After adjusting for age, sex, and smoking history, the OR was 0.770 (95\% CI 0.595-0.996).

\section{Subgroup analysis of the association between hOGGI rsI052I33 polymorphism and risk of NPC}

Among patients older than 40 years, individuals with genotype GG at rs 1052133 had 0.706 -fold risk of NPC
(95\% CI 0.524-0.950) compared to individuals with GC or CC (Table 3). After adjusting for sex and smoking history, the OR increased to 0.707 (95\% CI 0.524-0.953). Among women, those with GG had 0.571-fold risk of NPC (95\% CI 0.337-0.968) compared to those with GC or CC. After adjusting for age and smoking history, the OR was 0.574 (95\% CI 0.338-0.975). Among individuals with no smoking history, those with GG had 0.635 -fold risk of NPC (95\% CI 0.464-0.866) compared to those with GC or CC. After adjusting for sex and age, the OR was $0.634(95 \%$ CI 0.463-0.868).

\section{Association of hMUTYH rs3219472 polymorphism with risk of NPC}

No significant association was observed between hMUTYH rs3219472 genotype and risk of NPC (Table 2).

\section{Interactions between hOGGI rs I052I33} and hMUTYH rs3219472 SNPs in influencing risk of NPC

We examined all combinations of genotypes at both the hOGG1 rs1052133 and hMUTYH rs3219472 SNPs for possible joint effects on risk of NPC (Table 4). For this analysis,

Table 3 Distribution of hOGGI rsI052I33 genotypes in Chinese patients with nasopharyngeal carcinoma after stratification by age, sex, or smoking history

\begin{tabular}{|c|c|c|c|c|c|}
\hline \multirow[t]{2}{*}{ Subgroup } & \multirow[t]{2}{*}{$\mathbf{N}$ (case/control) } & \multicolumn{2}{|c|}{$\begin{array}{l}\text { Number of patients or controls } \\
\text { with genotype (case/control) }\end{array}$} & \multirow[t]{2}{*}{ OR $(95 \% \mathrm{Cl})$} & \multirow[t]{2}{*}{$\begin{array}{l}\text { Adjusted } O^{a} \\
(95 \% \mathrm{CI})\end{array}$} \\
\hline & & GG & $\mathbf{G C}+\mathbf{C C}$ & & \\
\hline \multicolumn{6}{|l|}{ Age (years) } \\
\hline$\leq 40$ & $|36 / 15|$ & $39 / 43$ & $97 / 108$ & $1.010(0.605-1.686)$ & $0.996(0.595-1.666)$ \\
\hline$>40$ & $352 / 422$ & $112 / 168$ & $240 / 254$ & $0.706(0.524-0.950)$ & $0.707(0.524-0.953)$ \\
\hline \multicolumn{6}{|l|}{ Sex } \\
\hline Male & $374 / 417$ & $|2| /|5|$ & $253 / 266$ & $0.842(0.627-|| 3 \mid)$. & $0.845(0.628-1.137)$ \\
\hline Female & $114 / 156$ & $30 / 60$ & $84 / 96$ & $0.57 \mid(0.337-0.968)$ & $0.574(0.338-0.975)$ \\
\hline \multicolumn{6}{|c|}{ Smoking history } \\
\hline Yes & $166 / 186$ & $53 / 53$ & $113 / 133$ & $0.850(0.539-1.340)$ & $0.857(0.543-1.354)$ \\
\hline No & $322 / 387$ & $98 / 158$ & $224 / 229$ & $0.635(0.464-0.866)$ & $0.634(0.463-0.868)$ \\
\hline
\end{tabular}

Notes: ${ }^{a}$ Calculated by multiple logistic regression after controlling for the other two stratifying factors. For example, the adjusted OR for different age subgroups was adjusted for sex and smoking history.

Abbreviations: hOGGI, human 8-oxoguanine DNA glycosylase I; OR, odds ratio; Cl, confidence interval. 
Table 4 Analysis of joint influence of genotypes at hOGGI rsI052I33 and hMUTYH rs3219472 on risk of nasopharyngeal carcinoma

\begin{tabular}{|c|c|c|c|c|c|}
\hline \multicolumn{2}{|l|}{ Genotype } & \multirow[t]{2}{*}{ NPC } & \multirow[t]{2}{*}{ Control } & \multirow[t]{2}{*}{$\mathbf{P}^{*}$} & \multirow{2}{*}{$\begin{array}{l}\text { Adjusted OR } \\
(95 \% \mathrm{Cl})\end{array}$} \\
\hline rs 1052133 & rs3219472 & & & & \\
\hline $\mathrm{CC}$ & AA & 13 & 6 & 1.00 & 1.00 \\
\hline GG & GG & 62 & 83 & 0.043 & $2.887(1.033-8.072)$ \\
\hline GG & GA & 70 & 101 & 0.026 & $3.183(1.147-8.835)$ \\
\hline GG & AA & 19 & 27 & 0.040 & $3.392(1.059-10.870)$ \\
\hline $\mathrm{CC}$ & GG & 34 & 37 & 0.111 & NS \\
\hline CC & GA & 34 & 42 & 0.065 & NS \\
\hline CG & GG & 119 & 126 & 0.096 & NS \\
\hline CG & AA & 31 & 39 & 0.062 & NS \\
\hline CG & $\mathrm{GA}$ & 106 & 112 & 0.098 & NS \\
\hline Total & & 488 & 573 & & \\
\hline
\end{tabular}

Notes: ${ }^{a}$ Calculated by multiple logistic regression after controlling for age, sex, and smoking history. ${ }^{*}$ Calculated using the chi-squared test with respect to the $P$-value associated with the combination of genotype CC at hOGGI rs I052I33 and genotype AA at hMUTYH rs3219472. NS means not shown due to $P>0.05$.

Abbreviations: hOGGI, human 8-oxoguanine DNA glycosylase I; hMUTYH, human MutY glycosylase homologue; NPC, nasopharyngeal carcinoma; OR, odds ratio; $\mathrm{Cl}$, confidence interval.

the combination of genotype CC at hOGG1 rs1052133 and genotype AA at hMYH rs3219472 (CC/AA) served as the reference. Individuals with genotype CC/AA were at significantly higher risk of NPC than those with other genotype combinations: the risk with CC/AA was 2.887 -fold higher than with GG/GG, 3.183-fold higher than with GG/GA, and 3.392-fold higher than with GG/AA. In contrast, genotype $\mathrm{CC} / \mathrm{AA}$ was associated with similar risk as genotypes $\mathrm{CC} / \mathrm{GG}, \mathrm{CC} / \mathrm{GA}, \mathrm{CG} / \mathrm{GG}, \mathrm{CG} / \mathrm{GA}$, and $\mathrm{CG} / \mathrm{AA}$.

\section{Discussion}

Our study of Chinese NPC patients from Guangxi Province and healthy controls indicates a significantly lower frequency of genotype GG at hOGG1 rs1052133 among patients, suggesting that this SNP is associated with susceptibility to NPC. Subgroup analyses further revealed a significantly lower frequency of GG in patients than controls among individuals older than 40 years, women, and those with no smoking history. These results provide the first evidence that genotype GG may help protect against NPC in certain populations.

Our observation of an association between hOGG1 rs1052133 and the risk of NPC is consistent with a previous report in Taiwan, suggesting that individuals with genotype GG or GC at this SNP are at 1.6-fold higher risk of NPC than those with genotype CC. ${ }^{10}$ On the other hand, these findings contrast with studies in patients from North African countries ${ }^{11}$ and Chinese patients from Chongqing, ${ }^{12}$ which failed to find a significant association between hOGG1 rs1052133 polymorphism and the risk of NPC. Ethnic and geographic differences may help explain these discrepancies, as well as the small size of the Chongqing study, ${ }^{12}$ which increases the risk of selective bias. Our findings should be confirmed in even larger studies looking at various ethnic groups in different locations, especially since our study was purely based on important clinical associations and did not take into account several possible novel confounders, such as body mass index. ${ }^{16}$

To our knowledge, this is the first study to evaluate the effect of hMUTYH rs3219472 SNP on the risk of NPC. Our results suggest no significant association between the two, contrasting with studies in Chinese patients reporting an association between genotype AA at this locus and elevated risk of cholangiocarcinoma, ${ }^{14}$ as well as an association between genotype GA and decreased risk of esophageal adenocarcinoma. ${ }^{15}$ When we examined possible joint effects of both SNPs hOGG1 rs1052133 and hMUTYH rs3219472 on the risk of NPC in our population, we found that subjects with genotype $\mathrm{CC}$ at hOGG1 rs1052133 and genotype AA at hMUTYH rs3219472 (CC/AA) were at significantly higher risk of NPC than those with other genotype combinations (GG/AA, GG/GG, and GG/GA). These results suggest that, at least in Asian populations, hMUTYH rs3219472 polymorphism may play a role in NPC susceptibility, just as it does in other malignancies. The fact that we observed this association only in combination with hOGG1 rs1052133 polymorphism highlights the multigenic nature of carcinogenesis and supports previous proposals that gene variants sometimes interact to affect cancer risk more than each gene on its own. ${ }^{12}$ It makes sense that perturbations in multiple steps of the BER pathway would affect the risk of NPC more than perturbations in a single step. At the same time, the fact that we observed the CC/AA genotype in such a low proportion of patients $(13 / 488)$ and controls (6/573) means that our results must be verified in larger, well-designed studies.

The molecular mechanism(s) by which hOGG1 rs1052133 polymorphism affects susceptibility to NPC are unknown. We found a significantly lower frequency of GG among NPC patients than controls, suggesting that this genotype protects against the disease. This is in contrast with the conclusion from several studies suggesting that genotype GG compromises the ability of hOGG1 to repair 8-oxoGs. Genotyping studies in patients from India, People's Republic of China, Japan, and other Asian countries have reported higher frequencies of genotypes GG or GC among patients with malignant tumors than among healthy controls, ${ }^{17,18}$ and laboratory studies have shown that the Ser326Cys hOGG1 variant encoded by genotype GG is more sensitive to inactivation by oxidative agents ${ }^{5,19}$ and shows nearly sevenfold 
lower repair activity ${ }^{20,21}$ than the Ser326 enzyme encoded by genotype CC. There are at least three possible explanations for the observed protective effect of genotype GG and previous studies reporting a deleterious effect.

First, the accumulation of DNA damage due to impaired hOGG1 activity may increase apoptotic cell death during cell division, which may protect against cancer in carriers of the mutant hOGG1 allele. ${ }^{22}$ This may explain why some studies have associated the G allele at hOGG1 rs1052133 with lower risk of breast and lung cancer. ${ }^{5,23}$ Recently, we have completed studies detecting the rs 1052133 SNP in some cell lines of NPC and normal immortalized nasopharyngeal epithelium. Interestingly, the results showed that genotypes of NPC cell lines, including CNE1, CNE2, 5-8F/6-10B, HK-1, HONE1, TWO3, and HNE1, were CC, and the normal epithelial cell lines NP69 and NP460 were GG (data not shown). These results may provide some clues discovering in vitro effect of rs 1052133 SNP on the NPC tumorigenesis. The following functional investigation using these cell lines would reveal a more precise mechanism by which this SNP may affect NPC carcinogenesis.

Second, the GG genotype at hOGG1 rs1052133 may reduce the repair of oxidative DNA damage only in the presence of excessive cellular oxidative stress. ${ }^{24}$ Indeed, our subgroup analyses revealed a protective effect of genotype GG specifically among those with no smoking history and among women, only $2.6 \%$ (3/114) of whom had a smoking history. No such protective effect was observed among individuals with a smoking history, and smoking is known to generate free radicals that induce oxidative and nitrative stress. ${ }^{17}$ These considerations lead us to propose that, in the presence of reasonable levels of oxidative stress, such as in nonsmokers, hOGG1 repair activity may not differ significantly between individuals with genotype GG or CC, such that genotype GG does not measurably affect the risk of NPC.

Third, the GG variant of rs 1052133 may affect NPC risk through other mechanisms. Other authors have suggested that this variant is linked to other functional polymorphisms in hOGG1 and/or other genes involved in repairing oxidative DNA damage. ${ }^{5}$ Future studies should aim to examine whether one or more of these three mechanisms operate in patients with NPC.

Besides a significant relation between SNP rs 1052133 and NPC risk, our study also indicates that the frequency of NPC patients with positive immunoglobulin A against the capsid antigen of EBV (EBV-VCA-IgA) is significantly higher than controls. This leads us to speculate that rs 1052133 SNP might affect the susceptibility to EBV infection in the cases in our cohort, and these two factors may have a joint effect on NPC risk. However, our stratified analyses showed no significant difference of $\mathrm{GG}$ or $\mathrm{CC}$ genotype frequencies between NPC and normal controls among those with positive EBV-VCA-IgA (data not shown), suggesting that rs 1052133 SNP may not affect the susceptibility to EBV infection. Our analyses also showed that GG or CC genotype frequencies were not significantly different between those with positive and negative EBV-VCA-IgA among the NPC cases (data not shown), indicating that the interaction between this SNPs and EBV infection may not significantly influence the NPC risk. However, considering the relatively low proportion of controls with positive EBV-related antibody (8/573) and the limited number of NPC cases (184/488) with negative EBV-related antibody, further investigations involving more such cases are necessary to obtain real information about the combined effect of EBV infection and SNPs in BER pathway on NPC risk.

Our conclusions should be treated with caution because of some important limitations of the present study. Its relatively small sample may lead to selective bias, affecting our results. Its cross-sectional design means that we cannot exclude that some controls will go on to develop NPC or other types of cancer, which would affect our findings.

\section{Conclusion}

Despite the limitations above, our study provides the first evidence that genotype GG at the hOGG1 rs1052133 SNP may be associated with decreased risk of NPC, especially in women, nonsmokers, and those older than 40 years. In addition, our data suggest that this hOGG1 SNP interacts with the hMUTYH rs3219472 SNP to influence the risk of NPC.

\section{Acknowledgments}

This study was supported by the Guangxi Natural Science Foundation of China (No. 2014GXNSFAA118187) and the National Natural Science Foundation of China (81360406 and 81460415).

\section{Disclosure}

The authors declare no conflicts of interest in this work.

\section{References}

1. Wei Z, Zeng X, Xu J, Duan X, Yang J, Xie Y. Prognostic value of the pretreatment serum level of cytokeratin fraction 21-1 in undifferentiated nasopharyngeal carcinoma: a study of 332 cases. Head Neck. 2014; 36(1):71-76.

2. Huang YJ, Zhang BB, Ma N, Murata M, Tang AZ, Huang GW. Nitrative and oxidative DNA damage as potential survival biomarkers for nasopharyngeal carcinoma. Med Oncol. 2011;28(1):377-384. 
3. Borrego S, Vazquez A, Dasi F, et al. Oxidative stress and DNA damage in human gastric carcinoma: 8-Oxo-7'8-dihydro-2'-deoxyguanosine (8-oxo-dG) as a possible tumor marker. Int J Mol Sci. 2013;14(2): 3467-3486.

4. Chen L, Elahi A, Pow-Sang J, Lazarus P, Park J. Association between polymorphism of human oxoguanine glycosylase 1 and risk of prostate cancer. J Urol. 2003;170(6 pt 1):2471-2474.

5. Liu CJ, Hsia TC, Tsai RY, et al. The joint effect of hOGG1 single nucleotide polymorphism and smoking habit on lung cancer in Taiwan. Anticancer Res. 2010;30(10):4141-4145.

6. Robertson AB, Klungland A, Rognes T, Leiros I. DNA repair in mammalian cells: base excision repair: the long and short of it. Cell Mol Life Sci. 2009;66(6):981-993.

7. Wang Y, Gao X, Wei F, et al. The hOGG1 Ser326Cys polymorphism contributes to digestive system cancer susceptibility: evidence from 48 case-control studies. Tumour Biol. 2015;36(2):1029-1038.

8. Yin ZB, Hua RX, Li JH, et al. Smoking and hOGG1 Ser326Cys polymorphism contribute to lung cancer risk: evidence from a meta-analysis. Tumour Biol. 2014;35(2):1609-1618.

9. Zhou C, Xie LP, Lin YW, Yang K, Mao QQ, Cheng Y. Susceptibility of XPD and hOGG1 genetic variants to prostate cancer. Biomed Rep. 2013;1(4):679-683.

10. Cho EY, Hildesheim A, Chen CJ, et al. Nasopharyngeal carcinoma and genetic polymorphisms of DNA repair enzymes XRCC1 and hOGG1. Cancer Epidemiol Biomarkers Prev. 2003;12(10):1100-1104.

11. Laantri N, Jalbout M, Khyatti M, et al. XRCC1 and hOGG1 genes and risk of nasopharyngeal carcinoma in North African countries. Mol Carcinog. 2011;50(9):732-737.

12. Li Q, Wang JM, Peng Y, et al. Association of DNA base-excision repair XRCC1, OGG1 and APE1 gene polymorphisms with nasopharyngeal carcinoma susceptibility in a Chinese population. Asian Pac J Cancer Prev. 2013;14(9):5145-5151.

13. Przybylowska K, Kabzinski J, Sygut A, Dziki L, Dziki A, Majsterek I. An association selected polymorphisms of XRCC1, OGG1 and MUTYH gene and the level of efficiency oxidative DNA damage repair with a risk of colorectal cancer. Mutat Res. 2013;74(5-746):6-15.

14. You SH, Wang X, Huang S, et al. MYH rs3219476 and rs3219472 polymorphisms and risk of cholangiocarcinoma. Mol Med Rep. 2013;7(1): $347-351$.
15. Kong F, Han XY, Luan Y, et al. MUTYH association with esophageal adenocarcinoma in a Han Chinese population. Asian Pac J Cancer Prev. 2013;14(11):6411-6413.

16. Luo H, Li Z, Qing Y, et al. Single nucleotide polymorphisms of DNA base-excision repair genes (APE1, OGG1 and XRCC1) associated with breast cancer risk in a Chinese population. Asian Pac JCancer Prev. 2014; 15(3):1133-1140.

17. Kumar A, Pant MC, Singh HS, Khandelwal S. Role of OGG1 Ser326Cys polymorphism and 8-oxoguanine DNA damage in risk assessment of squamous cell carcinoma of head and neck in North Indian population. Mutat Res. 2011;726(2):227-233.

18. Guan P, Huang D, Yin Z, Zhou B. Association of the hOGG1 Ser326Cys polymorphism with increased lung cancer susceptibility in Asians: a meta-analysis of 18 studies including 7592 cases and 8129 controls. Asian Pac J Cancer Prev. 2011;12(4):1067-1072.

19. Moritz E, Pauly K, Bravard A, Hall J, Radicella JP, Epe B. hOGG1Cys326 variant cells are hypersensitive to DNA repair inhibition by nitric oxide. Carcinogenesis. 2014;35(6):1426-1433.

20. Takezaki T, Gao CM, Wu JZ, et al. hOGG1 Ser(326)Cys polymorphism and modification by environmental factors of stomach cancer risk in Chinese. Int J Cancer. 2002;99(4):624-627.

21. Kohno T, Shinmura K, Tosaka M, et al. Genetic polymorphisms and alternative splicing of the hOGG1 gene, that is involved in the repair of 8-hydroxyguanine in damaged DNA. Oncogene. 1998;16(25): 3219-3225.

22. Hung RJ, Hall J, Brennan P, Boffetta P. Genetic polymorphisms in the base excision repair pathway and cancer risk: a HuGE review. Am J Epidemiol. 2005;162(10):925-942.

23. Yuan W, Xu L, Feng Y, et al. The hOGG1 Ser326Cys polymorphism and breast cancer risk: a meta-analysis. Breast Cancer Res Treat. 2010; 122(3):835-842.

24. Lee AJ, Hodges NJ, Chipman JK. Interindividual variability in response to sodium dichromate-induced oxidative DNA damage: role of the Ser326Cys polymorphism in the DNA-repair protein of 8-oxo-7,8dihydro-2'-deoxyguanosine DNA glycosylase 1. Cancer Epidemiol Biomarkers Prev. 2005;14(2):497-505.
OncoTargets and Therapy

\section{Publish your work in this journal}

OncoTargets and Therapy is an international, peer-reviewed, open access journal focusing on the pathological basis of all cancers, potential targets for therapy and treatment protocols employed to improve the management of cancer patients. The journal also focuses on the impact of management programs and new therapeutic agents and protocols on
Dovepress

patient perspectives such as quality of life, adherence and satisfaction The manuscript management system is completely online and includes a very quick and fair peer-review system, which is all easy to use. Visit http://www.dovepress.com/testimonials.php to read real quotes from published authors. 